\title{
Adults who were born preterm with a very low birth weight reported a similar health-related quality of life to their term-born peers
}

\author{
*Johan Björkqvist, $\mathrm{MD}^{1,2}$, Petteri Hovi, MD, $\mathrm{PhD}^{1,2}$, Anu-Katriina Pesonen, $\mathrm{PhD}^{3}$, Katri Räikkönen, $\mathrm{PhD}^{3}$, Kati \\ Heinonen $\mathrm{PhD}^{3}$, Anna-Liisa Järvenpää, MD, $\mathrm{PhD}^{2}$, Johan G. Eriksson, MD, $\mathrm{DMSc}^{4-7}$, Sture Andersson, MD, \\ $\mathrm{PhD}^{2}$, Eero Kajantie, MD, DMSc ${ }^{1,2,8}$.
}

${ }^{1}$ National Institute for Health and Welfare, Department of Public Health Solutions, Finland

${ }^{2}$ Children's Hospital, University of Helsinki and Helsinki University Hospital, Finland

${ }^{3}$ Department of Psychology and Logopedics, Faculty of Medicine, University of Helsinki, Finland

${ }^{4}$ National Institute for Health and Welfare, Department of Chronic Disease Prevention, Helsinki, Finland

${ }^{5}$ Department of General Practice and Primary Health Care, University of Helsinki, and Helsinki University Hospital, Helsinki, Finland

${ }^{6}$ Vasa Central Hospital, 65130 Vasa, Finland

${ }^{7}$ Folkhälsan Research Centre, Helsinki, Helsingfors Universitet, Helsinki, Finland

${ }^{8}$ PEDEGO Research Unit, MRC Oulu, Oulu University Hospital and University of Oulu, Oulu, Finland

Short title: Health-related quality of life among adults born preterm

${ }^{*}$ Corresponding author at National Institute for Health and Welfare, Department of Public Health Solutions, PL 30, 00271 Helsinki, Finland. Tel.: +35829 524 6000. E-mail address: johan.bjorkqvist@helsinki.fi 
Approximately $1 \%$ of European infants are born preterm with a very low birth weight (VLBW) of less than $1,500 \mathrm{~g}$ and the first babies born at the threshold of viability who received modern neonatal intensive care are now adults. While most live healthy lives, average VLBW adults tend to have more neurodevelopmental disabilities, lower educational achievement and higher rates of chronic conditions (1). Health-related quality of life (HRQoL) is defined as a construct of physical, mental and social wellbeing. Previous studies have suggested that differences and changes in quality of life and HRQoL between preterm and term groups are small, are not necessarily observed by self-reports and diminish over time (2,3). However, four longitudinal studies from 2015 and 2016 (4-7) challenged this view by showing constantly lower levels of HRQoL in adults born very or extremely preterm, before 32 weeks or 28 weeks of gestation, respectively, or with an extremely low birth weight of less than $1,000 \mathrm{~g}$. This even applied to adults who were not impaired $(4,6)$. Although many of the outcomes of preterm birth differ by sex (8) or intrauterine growth (9), few studies have assessed whether those two factors also have an impact on HRQoL and that was the aim of our study. We assessed differences in overall HRQoL and in a 15-dimensional profile between adults born preterm and those born at term.

The Helsinki Study of Very Low Birth Weight Adults is a birth cohort of all 335 VLBW infants born in 1978-1985, who were treated at the only neonatal intensive care unit in Uusimaa, Finland. The controls are term-born infants who were not small for gestational age (SGA) and were groupmatched for sex, age and birth hospital. SGA status served as proxy for intrauterine growth restriction and was defined as a birth weight of less than -2 standard deviations (SD) for sex and age, as opposed to appropriate for gestational age (AGA). At 18 to 27 years of age, 166 VLBW adults, $65.1 \%$ of those invited, and 172 controls, $54.8 \%$ of those invited, were clinically examined. The design and characteristics of the Helsinki Study have previously been described (10). There were 21 VLBW participants with at least one neurosensory impairment (NSI): 14 had cerebral palsy, five had a developmental disorder and five had a severe visual impairment. 15D, which is a generic 15-dimensional, self-administered instrument that measures HRQoL (www.15d- 
instrument.net) was completed by $336 / 338$ members of the cohort: 110 born AGA VLBW, 54 born SGA VLBW and 172 term-born controls.

Participants estimated their performance by scoring 15 health areas on an ordinal level of $1-5$, for example one for normal hearing and five for total deafness (11). The dimensions and valuations of the questionnaire were developed with feedback from healthcare staff and patients (11). Up to three missing answers can be predicted using Sintonen's algorithm and this happened in three cases for one missing answer. One participant had four missing answers, so her score could not be calculated, leaving us with 335 questionnaires for the analysis. The 1-5 levels were transformed into $0.000-1.000$ values to generate a 15 -dimensional profile and then, with the use of preference weights, into a single aggregate 15D score, with higher values being better and lower being worse. When the $15 \mathrm{D}$ score is compared between groups, the minimum important difference is \pm 0.015 , provided that the difference is statistically significant. This score indicates slightly better or worse, while \pm 0.035 indicates much better or worse (12).

We used the Mann-Whitney $U$ test to compare the 15D scores, due to their non-normal distribution. We also used linear regression, with the rank of the 15D score as the outcome, and this was adjusted for sex, gestational age group, parental education and NSI. It was also used to assess the interaction of sex.

The Helsinki University Central Hospital Ethics Committee for Gynaecology, Obstetrics, Pediatrics and Psychiatry approved the study and all participants provided written, informed consent.

The clinical characteristics of the cohort are shown in Table S1 and Table S2 displays the central 15D comparisons. We report the 15D score and the 15-dimensional profile for the following comparisons: men and women, the whole VLBW group and controls and the AGA VLBW, SGA VLBW and control groups (Figure 1A). We also compared the three groups by sex (Figures 1B and $1 \mathrm{C})$.

On average, women reported a lower $15 \mathrm{D}$ score than men $(\Delta 0.034, p<0.001)$. In the profile, women reported significantly lower in all areas except mobility, hearing, eating, speech, usual activities and sexual activity (data not shown). 
There was no difference in the total 15D score between the whole VLBW group and control group ( 0.930 versus $0.931, p=0.85)$ and no significant differences in any of the profile dimensions. The $21 \mathrm{NSI}$ participants did not have a significantly lower 15D score than the control group, but they did score lower on mobility, vision, sexual activities and eating (data not shown).

Figure 1 shows the 15D scores and profiles based on relative birth weight. The SGA VLBW group reported a significantly lower $15 \mathrm{D}$ score than the AGA VLBW group $(0.911$ versus $0.939, \Delta 0.028, p$ $=0.039)$ (Figure 1A). The AGA VLBW group scored significantly lower on mobility and significantly higher on depression than the controls. The SGA VLBW group scored significantly lower than the controls on eating and significantly lower than the AGA VLBW group on mental functions, depression and vitality (Figure 1A).

Figures $1 \mathrm{~B}$ and $1 \mathrm{C}$ show the three groups of relative birth weight by sex. There was no significant difference in the 15D score among men, but AGA VLBW men scored significantly lower on mobility and higher on speech, mental functions and depression than the control men. The SGA VLBW men did not differ significantly from either group. The AGA VLBW women had similar scores as the control women in all dimensions, while the SGA VLBW women reported a significantly lower 15D score than both the control women $(0.886$ versus $0.922, \Delta 0.036, p=0.018)$ and the AGA VLBW women $(0.886$ versus $0.923, \Delta 0.037, p=0.025)$ (Figure $1 \mathrm{C})$. In the profile, the SGA VLBW women reported significantly lower scores than the other two groups on eating, mental functions and depression. Furthermore, the SGA VLBW women reported significantly lower scores than the controls on speech and lower scores than the AGA VLBW women on excretion and vitality. Our regression analysis showed that parental education and NSI were unrelated to the $15 \mathrm{D}$ score and adjusting for them had no effect on the results. The $p$ values for sex interaction were 0.07 for the SGA VLBW versus control group and 0.28 for the AGA VLBW versus control group.

We found no significant differences in overall HRQoL between the whole VLBW group and controls. However, the HRQoL of the SGA VLBW group was significantly lower than the AGA VLBW group and this exceeded the minimum important difference. It seemed to be caused by two 
trends: the significantly lower HRQoL among SGA VLBW women and AGA VLBW men reporting higher scores in several dimensions.

Our findings could partly shed light on some of the discrepancies in previous studies that compared HRQoL between adults born preterm and term-born controls. Our subgroup analysis revealed sex-related differences between the groups that were consistent with previous findings. We found that SGA VLBW women experienced poorer mental function and more depressive symptoms. More withdrawal, anxiety, depression and lower social competence has been reported among extremely preterm girls (13) and lower scores on scales for mental health, social functioning and emotional role has been found among term-born SGA participants (14). The lower mobility scores in our study were consistent with studies that have shown lower levels of physical functioning, especially among male subjects born preterm (15).

The strengths of this study were the well-described cohort of VLBW subjects and controls and the use of a feasible, reliable, valid and sensitive questionnaire (11).

We have previously discussed the limitations of the Helsinki Study of Very Low Birth Weight Adults (10). The NSI participants were unlikely to have been the cause of our main findings, because they reported lower scores that were, on the whole, different to the low scoring areas reported by the SGA women. The NSI participants were less likely to participate and this means that we may have overestimated the HRQoL in the VLBW population (11). In order to minimise the participation bias, the NSI participants were not excluded from the study. Another possible limitation in interpretation was the absence of term SGA participants. It is possible that the poorer scores among SGA VLBW women might have been a cohort-specific characteristic (9), although other studies have reported similar findings (13). While our study had adequate power to compare overall HRQoL, comparisons of individual dimensions may have included chance findings and should be treated with caution. The questionnaire had not previously been used to assess the HRQoL of subjects born preterm, so the results are not directly comparable with studies using other instruments. It is encouraging that young adults born preterm reported similar overall HRQoL to term-born controls. Because neonatal care has evolved since the 1980s, our findings do not reveal the 
effects of modern treatment on HRQoL, but they are directly relevant to VLBW survivors, now in their 30s and 40s, who already number nearly one million people, just in Europe. 


\section{Finance statement}

This study was financed by: the Academy of Finland, the Finnish Foundation for Pediatric Research, the Finnish Special Governmental Subsidy for Health Sciences, the Jalmari and Rauha Ahokas Foundation, the Juho Vainio Foundation, the Novo Nordisk Foundation, the Päivikki and Sakari Sohlberg Foundation, the University of Helsinki, the Perklén Foundation, the Signe and Ane Gyllenberg Foundation, the Sigrid Juselius Foundation, the Yrjö Jahnsson Foundation, the Finnish Medical Foundation, the Emil Aaltonen Foundation and Finska Läkaresällskapet.

\section{Conflicts of interests.}

The authors have no conflicts of interest to declare.

\section{Abbreviations}

HRQoL, health-related quality of life; VLBW, very low birth weight; SGA, small for gestational age; AGA, appropriate for gestational age; NSI, neurosensory impairment.

\section{References}

1. Raju TNK, Buist AS, Blaisdell CJ, Moxey-Mims M, Saigal S. Adults born preterm: a review of general health and system-specific outcomes. Acta Paediatr 2017; 106: 1409-1437.

2. Zwicker JG, Harris SR. Quality of life of formerly preterm and very low birth weight infants from preschool age to adulthood: a systematic review. Pediatrics 2008; 121: e366-76.

3. van Lunenburg A, van der Pal SM, van Dommelen P, van der Pal - de Bruin KM, Bennebroek Gravenhorst J, Verrips GHW. Changes in quality of life into adulthood after very preterm birth and/or very low birth weight in the Netherlands. Health Qual Life Outcomes 2013; 11: 51.

4. Båtsvik B, Vederhus BJ, Halvorsen T, Wentzel-Larsen T, Graue M, Markestad T. Health-related quality of life may deteriorate from adolescence to young adulthood after extremely preterm birth. Acta Paediatr 2015; 104: 948-55. 
5. Baumann N, Bartmann P, Wolke D. Health-related quality of life into adulthood after very preterm birth. Pediatrics 2016; 137: e20153148.

6. Saigal S, Ferro MA, van Lieshout RJ, Schmidt LA, Morrison KM, Boyle MH. Health-related quality of life trajectories of extremely low birth weight survivors into adulthood. J Pediatr 2016; 179: $68-73$.

7. Husby IM, Stray KM, Olsen A, Lydersen S, Indredavik MS, Brubakk AM et al. Long-term followup of mental health, health-related quality of life and associations with motor skills in young adults born preterm with very low birth weight. Health Qual Life Outcomes 2016; 14: 56.

8. Hovi P, Vohr B, Ment LR, Doyle LW, McGarvey L, Morrison KM et al. Blood pressure in young adults born at very low birth weight: Adults born Preterm International Collaboration. Hypertension 2016; 68: 880-7.

9. Räikkönen K, Pesonen AK, Heinonen K, Kajantie E, Hovi P, Järvenpää AL et al. Depression in young adults with very low birth weight: the Helsinki study of very low-birth-weight adults. Arch Gen Psychiatry 2008; 65: 290-6.

10. Hovi P, Andersson S, Eriksson JG, Järvenpää A, Strang-Karlsson S, Mäkitie O et al. Glucose Regulation in Young Adults with Very Low Birth Weight. N Engl J Med 2007; 356: 2053-63.

11. Sintonen $\mathrm{H}$. The 15D instrument of health-related quality of life: properties and applications. Ann Med 2001; 33: 328-36.

12. Alanne S, Roine RP, Räsänen $P$, Vainiola $T$, Sintonen $H$. Estimating the minimum important change in the 15D scores. Qual Life Res 2015; 24: 599-606.

13. Vederhus BJ, Eide GE, Natvig GK, Markestad T, Graue M, Halvorsen T. Health-related quality of life and emotional and behavioral difficulties after extreme preterm birth: developmental trajectories. PeerJ 2015; Jan 20; 3: e738. 
14. Lund LK, Vik T, Lydersen S, Løhaugen GC, Skranes J, Brubakk AM et al. Mental health, quality of life and social relations in young adults born with low birth weight. Health Qual Life Outcomes 2012; 10: 146.

15. Baumgardt M, Bucher HU, Mieth RA, Fauchère JC. Health-related quality of life of former very preterm infants in adulthood. Acta Paediatr 2012; 101: 59-63.

Table S1 Characteristics of the control group, VLBW group and VLBW subgroups.

Table S2 Mean values and comparisons of subgroups. 
Legend for Figure 1.

$$
\begin{aligned}
& \mathbf{-}-\text { = Controls } \quad \mathbf{Q 0}=\text { significant difference between controls and AGA VLBW, } \mathrm{p}<0.05 \\
& \text {-0.- = AGA VLBW } \quad 0 \text { = significant difference between AGA VLBW and SGA VLBW, } p<0.05 \\
& \text {. } \circ \cdots=\text { SGA VLBW } \quad \square \bigcirc=\text { significant difference between controls and SGA VLBW, } p<0.05
\end{aligned}
$$

Figure 1. 15D score and profiles in young adulthood of controls, AGA VLBW and SGA VLBW.

Figure 1A, all participants: controls $n=172$, AGA VLBW $n=109$, SGA VLBW $n=54$.

Figure 1B, men: controls $n=69$, AGA VLBW $n=48$, SGA VLBW $n=22$.

Figure 1C, women: controls $n=103$, AGA VLBW $n=61$, SGA VLBW $n=32$.

$\mathrm{AGA}=$ appropriate for gestational age, $\mathrm{SGA}=$ small for gestational age, VLBW = very low birth weight. One missing answer was replaced for three participants. The 15D score could not be calculated for one participant in the AGA VLBW group who had four missing answers.

Brief description of the 15 dimensions according to best answer:

Mobility - walking without aid. Vision - normal reading or watching tv. Hearing - normal hearing. Breathing - no shortness of breath or other breathing difficulty. Sleeping - no problems with sleeping. Eating - ability to eat independently. Speech - ability to speak clearly, audibly, and fluently. Excretion - bladder and bowel works without problems. Usual activities - ability to perform usual activities without difficulty. Mental functions - clear thinking and function of memory.

Discomfort - no pain, ache, nausea, itching etc. Depression - no feelings of sadness, melancholia, or depression. Distress - no feelings of anxiousness, stress, or nervousness. Vitality - feeling of health and energy. Sexual activity - health does not have impact on sexual activity. 


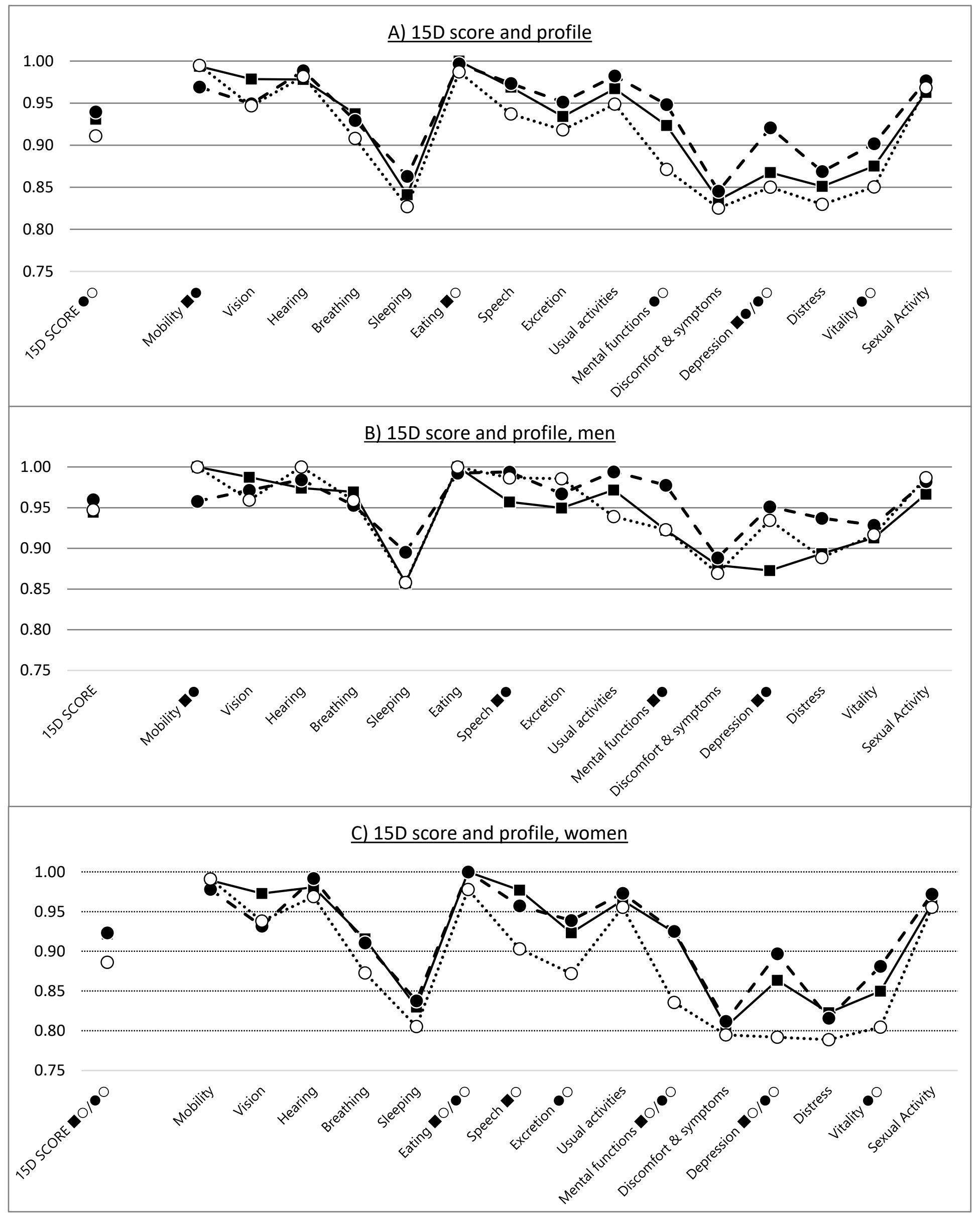

